\title{
Video Article \\ Robot-Assisted Radical Antegrade Modular Pancreatosplenectomy Including Resection and Reconstruction of the Spleno-Mesenteric Junction
}

\author{
Niccolò Napoli ${ }^{1}$, Emanuele F. Kauffmann ${ }^{1}$, Francesca Menonna ${ }^{1}$, Sara lacopi ${ }^{1}$, Concetta Cacace ${ }^{1}$, Ugo Boggi ${ }^{1}$ \\ ${ }^{1}$ Division of General and Transplant Surgery, University of Pisa
}

Correspondence to: Ugo Boggi at u.boggi@med.unipi.it

URL: https://www.jove.com/video/60370

DOI: doi: $10.3791 / 60370$

Keywords: Medicine, Issue 155, robot, robotic, robot-assisted, minimally invasive, laparoscopy, laparoscopic, distal pancreatectomy, distal splenopancreatectomy, pancreatic cancer, radical antegrade modular pancreatosplenectomy, RAMPS

Date Published: $1 / 3 / 2020$

Citation: Napoli, N., Kauffmann, E.F., Menonna, F., lacopi, S., Cacace, C., Boggi, U. Robot-Assisted Radical Antegrade Modular Pancreatosplenectomy Including Resection and Reconstruction of the Spleno-Mesenteric Junction. J. Vis. Exp. (155), e60370, doi:10.3791/60370 (2020).

\section{Abstract}

This article shows the technique of robot-assisted radical antegrade modular pancreatosplenectomy, including resection and reconstruction of the spleno-mesenteric junction, for cancer of the body-tail of the pancreas. The patient is placed supine with the legs parted and a pneumoperitoneum is established and maintained at $10 \mathrm{mmHg}$. To use the surgical system, four $8 \mathrm{~mm}$ ports and one $12 \mathrm{~mm}$ port are required. The optic port is placed at the umbilicus. The other ports are placed, on either side, along the pararectal line and the anterior axillary line at the level of the umbilical line. The assistant port $(12 \mathrm{~mm})$ is placed along the right pararectal line. Dissection begins by detaching the gastrocolic ligament, thus opening the lesser sac, and by a wide mobilization of the splenic flexure of the colon. The superior mesenteric vein is identified along the inferior border of the pancreas. Lymph node number $8 \mathrm{a}$ is removed to permit clear visualization of the common hepatic artery. A tunnel is then created behind the neck of the pancreas. To permit safe resection and reconstruction of the spleno-mesenteric junction, further preemptive dissection is required before dividing the pancreatic neck to bring in clear view all relevant vascular pedicles. Next, the splenic artery is ligated and divided, and the pancreatic neck is divided, with selective ligature of the pancreatic duct. After vein resection and reconstruction, dissection proceeds to complete the clearance of peripancreatic arteries that are peeled off from all lympho-neural tissues. Both celiac ganglia are removed en-bloc with the specimen. The Gerota fascia covering the upper pole of the left kidney is also removed en-bloc with the specimen. Division of short gastric vessels and splenectomy complete the procedure. A drain is left near the pancreatic stump. The round ligament of the liver is mobilized to protect the vessels.

\section{Video Link}

The video component of this article can be found at https://www.jove.com/video/60370/

\section{Introduction}

The incidence and mortality of pancreatic cancer are increasing, and the disease will soon become the second leading cause of cancer related death in Western countries ${ }^{1}$. The high fatality rate of pancreatic cancer is mostly related to the biological aggressiveness of this tumor type, with early and rapid metastatic dissemination ${ }^{2}$. For this reason, only approximately $20 \%$ of the patients are diagnosed with a seemingly localized disease. In these patients, radical tumor resection, in association with either neoadjuvant ${ }^{3,4}$ or adjuvant chemotherapy ${ }^{5}$, provides the only hope for a cure.

The diagnosis of pancreatic cancer located in the body-tail of the pancreas is often made when the tumor has already grown extensively or metastases are evident ${ }^{6,7}$. The few patients with a seemingly localized disease are those who could benefit from surgery, especially if negative resection margins are achieved ${ }^{8}$ and an adequate number of lymph nodes is retrieved ${ }^{9}$. Patients meeting these criteria could actually attain longterm survival, as left-sided pancreatic cancers are associated with a less aggressive malignant phenotype when compared with pancreatic head cancers $^{10}$.

Radical antegrade modular pancreatosplenectomy (RAMPS), first described by Strasberg et al. ${ }^{11}$, is a procedure that was specifically conceived to provide radical resection of pancreatic cancers located in the body-tail. Although laparoscopic RAMPS was shown to be feasible in wellselected patients ${ }^{12}$, the complexity of this procedure and the high rate of margin negative resection reported after robotic procedures ${ }^{13}$ suggest that robotic assistance could be rewarding in this operation. We herein describe the technique for robotic assisted RAMPS, that was developed in a center with experience in thousands of robotic procedures and in over 350 robotic pancreatic resections.

\section{Protocol}

The procedure described herein was conducted in compliance with the guidelines set out by the Ethics Committee of Pisa University Hospital for robotic operations, including regulations on research activity. 
NOTE: The patient is a 70-year-old female with a $3 \mathrm{~cm}$ pancreatic ductal adenocarcinoma located in the body of the pancreas close to the neck of the gland. The patient presented with abdominal pain. Her past medical history demonstrated arterial hypertension and appendectomy. Totalbody contrast-enhanced computed tomography (CT) showed a hypoenhancing pancreatic tumor strictly adherent to the spleno-mesenteric junction, with associated upstream dilation of the main pancreatic duct (Figure 1). No distant metastasis was identified making the tumor potentially resectable with curative intent.

\section{Experimental pre-operation}

1. Patient selection

1. Establish a diagnosis of pancreatic cancer, by either biopsy or unequivocal imaging findings.

2. Rule out distant metastasis by total-body contrast-enhanced CT scan. Perform the scan within 4 weeks of surgery ${ }^{14}$.

3. Assay tumor markers (CEA and Ca 19.9).

NOTE: High preoperative levels of Ca 19.9 have prognostic implications ${ }^{15}$, but low levels do not call into doubt the indication for surgery ${ }^{16}$.

4. Ensure that the patient is fit for surgery and eligible for a minimally invasive approach ${ }^{17,18}$

5. Do not perform RAMPS during the learning curve ${ }^{19}$.

6. Do not accept patients with tumors clearly involving the large peripancreatic vessels, until proficiency is achieved with standard RAMPS.

2. Patient preparation

1. Provide standard pre-surgical preparation.

2. Provide vaccination against encapsulated bacteria (Streptococcus pneumoniae, Neisseria meningitidis, Haemophilus influenza type B) to prevent overwhelming post-splenectomy sepsis ${ }^{20}$.

3. Equipment

1. Ensure the availability of a robotic system.

NOTE: So far, only one robotic system (Table of Materials) has been used for pancreatic resections ${ }^{18}$. Herein a last generation robotic system is used. The docking technique and the targeting procedure are those specific to this system.

2. Ensure that standard laparoscopic equipment and the following robotic instruments are available: small and medium hem-o-lok clip appliers, Maryland bipolar forceps, monopolar curved scissors, harmonic shears, and large needle drivers.

3. In case of vein resection, ensure that the following instruments are available: robotic black diamond micro forceps and laparoscopic bulldog clamps.

4. Ensure that all necessary sutures and consumables (Table of Materials) are available.

5. Ensure that a laparoscopic stapler is available.

\section{Surgical preparation}

1. Anesthesia ${ }^{17}$

1. Assess operative risk by providing a grade according to the American Society of Anesthesiologists' (ASA) classification of Physical Health.

2. Place at least one large bore (14 G or $16 \mathrm{G}$ ) intravenous cannula in a peripheral vein. Place a central venous line in patients with limited possibility of peripheral vein cannulation.

3. Monitor electrocardiogram, arterial pressure (cannulation of radial artery), capnography, pulse oximetry, urinary volumes, and body temperature.

4. Provide general anesthesia.

NOTE: Both inhalation and intravenous anesthesia can be used.

5. Provide deep neuromuscular blockade (rocuronium bromide: $0.075-0.1 \mathrm{mg} / \mathrm{kg}$ ).

6. Insert a nasogastric tube.

7. During surgery, perform blood gas analysis to verify blood gases and $\mathrm{pH}$.

8. At the end of the procedure, reverse anesthesia and remove the nasogastric tube.

2. Operation setting

NOTE: A schematic view of the operating room setup is provided in Figure 2.

1. Have the main surgeon operate from the robotic console.

2. Have a laparoscopic surgeon (first assistant) stand between the patient's legs. He or she operates suction, introduces and withdraws sutures, helps with retraction, and fires staplers.

3. Have an assistant surgeon stand on the left side of the patient. He or she exchanges robotic instruments and assists the laparoscopic surgeon.

4. Have a scrub nurse stand on the right side of the patient.

5. Place the patient supine, with the legs parted (French position) on an operating table equipped with a thermic blanket (Figure 3A).

6. Place intermittent pneumatic compression cuffs around the legs (Figure 3B), for prophylaxis of deep vein thrombosis.

7. Secure the patient to the operating table with wide bandings (Figure $3 \mathbf{C}$ ).

8. Prep the patient as to widely expose the abdomen (Figure 3D). Include the suprapubic region to permit a Pfannenstiel incision for specimen extraction.

NOTE: For all the other aspects the patients have to be prepared as for major laparoscopic surgery in cooperation with the anesthesia team $^{17}$. 


\section{Preparatory surgical maneuvers and docking of the robotic system}

1. Establish a pneumoperitoneum, using either a Veress needle or an open technique. Maintain pneumoperitoneum at approximately $10 \mathrm{mmHg}$.

2. Place the $8 \mathrm{~mm}$ robotic camera port just below or just above the umbilicus, depending on individual abdominal configuration. NOTE: The camera port should be approximately $10-15 \mathrm{~cm}$ from the closest boundary of the target anatomy.

3. Insert the robotic laparoscope and explore the abdomen searching for occult metastatic deposits. Biopsy any identified nodule and send it for frozen section histology. If no metastasis is discovered, place the other ports.

4. Place all ports along the transverse umbilical line. Place the $12 \mathrm{~mm}$ assistant port along the right pararectal line. Place the remaining robotic ports along the anterior axillary line, on either sides, and along the left pararectal line (Figure 4).

NOTE: Ideal port spacing is 6-8 cm. A minimum space of $4 \mathrm{~cm}$ can be accepted. Ensure $2 \mathrm{~cm}$ space between ports and bony prominences.

5. Adjust the operating table in reverse Trendelenburg position $\left(15-20^{\circ}\right)$ and tilt it towards the patient's right side $\left(5-8^{\circ}\right)($ Figure 5$)$. Position the robotic tower where staff will not be walking or standing to maximize patient access from the bedside.

6. To begin docking, align the laser crosshair of the boom over the camera port (Figure 6A). Use robotic arm number 2 for the camera.

7. Direct the camera arm between $L$ and $E$ on the FLEX icon at the base of the robotic arm (Figure 6B).

8. Clutch and point the camera to target the operative anatomy (Figure $6 \mathrm{C}$ ). Execute targeting by pressing the dedicated button on the camera head.

NOTE: Targeting automatically adjusts height, translation, and rotation of the overhead boom to maximize the range of motion of the robotic arms. The remaining arms are docked (Figure 6D), and the robotic instruments are inserted under vision.

\section{Pancreatectomy}

1. Open the lesser sac by dividing the reflection of colon and omentum. Do not go through the gastrocolic ligament as this could result in omental infarction ${ }^{21}$.

2. Start dissection midway along the transverse mesocolon and extend to the right until the hepatic flexure of the colon is reached, and to the left until the splenic flexure of the colon is fully mobilized. Once the lesser sac is fully open, the pancreatic body and tail become clearly visible.

3. Begin dissection of the peritoneum along the inferior margin of the pancreas, to allow mobilization of the body-tail of the pancreas.

4. Identify the superior mesenteric vein. NOTE: The superior mesenteric vein is a key landmark to proceed safely with further dissections.

5. In preparation for the creation of a tunnel behind the pancreatic neck, identify the common hepatic artery and the portal vein above the pancreatic neck. Resect lymph node number $8 \mathrm{~A}$ to bring the common hepatic artery in clear view.

6. Seal as many lymphatic vessels as possible using either hem-o-lok clips or ligatures. Once the course of the common hepatic artery is clearly defined, dissect the lymphatic tissue laying between the artery and the superior margin of the pancreatic neck to bring the portal vein in clear view.

7. Tag the common hepatic artery with a vessel loop to increase visibility and facilitate handling of the vessel during the procedure.

8. Perform dissection around the major arteries using cold scissors as the use of energy devices may result in thermal injury to the vessel walls, thus potentially increasing the risk of delayed bleeding ${ }^{22}$. Peel off the common hepatic artery, the celiac trunk and the first portion of the splenic artery by the surrounding lympho-neural tissues to have a clear picture of the vascular anatomy.

NOTE: In the accompanying video, an injury occurs to the dorsal pancreatic artery. The bleeding was fixed with a 5/0 polypropylene suture. Ligature and division of the dorsal pancreatic arteru would have been required anyway as this maneauver improves exposure of the origin of the splenic artery and offers more room for safe ligature of this large artery.

9. Divide the splenic artery between ligatures or clips. Apply two ligatures proximally and divide the vessel between two hem-o-lok clips. Whenever possible, divide the splenic artery before dividing the splenic vein, as this prevents the occurrence of sinistral portal hypertension, thus reducing blood pooling in the spleen and the amount of backwards bleeding.

1. Alternatively, use a stapler, loaded with a vascular cartridge.

NOTE: A tunnel behind the neck of the pancreas is developed at this stage. However, as suspected at pre-operative imaging, the tumor was strictly adherent to the spleno-mesenteric junction, making it preferable to further mobilize the specimen in order to achieve wider control of all vascular pedicles, before proceeding with vein resection and reconstruction.

10. Identify the superior mesenteric artery, to the left side of the superior mesenteric vein. Peel off the superior mesenteric artery $180^{\circ}$ on its left aspect.

11. Identify the inferior mesenteric artery and save to be used as a vascular patch at the time of vein reconstruction. During perivascular dissections, clip large lymphatics to reduce the amount of lymphatic leak.

12. Begin dissection medial to lateral in a posterior plane to remove a large amount of the retroperitoneal soft tissue en-bloc with the specimen. Identify the left adrenal gland during this stage. Further to the left, remove the Gerota fascia covering the upper pole of the left kidney enblock with the specimen, thus uncovering the anterior surface of the upper renal pole. The left renal vein and the left adrenal vein are clearly identified.

13. Divide the inferior mesenteric vein between clips. Spare a segment of the vein for vascular reconstruction. Dissect the splenic vein free proximal to the site of the tumor adherence to achieve upstream vascular control.

14. Place a transfix suture at the inferior margin of the gland to occlude the transverse pancreatic artery. Divide the neck of the pancreas. When enough room is available, use a laparoscopic or robotic stapler. Alternatively, divide the neck using harmonic scissors.

NOTE: For the patient shown in the video, the pancreas was divided using harmonic shears because of the limited space available.

15. Identify, dissect, and ligate the main pancreatic duct. Close the transection surface in a fish-mouth configuration using interrupted sutures of 4/0 expanded polytetrafluoroethylene (e-PTFE).

16. When possible, send the pancreatic margin for frozen section histology. Delay freezing the section of pancreatic margin after specimen extraction if the margin is perceived to be so close to the tumor that intracorporeal sampling appears troublesome.

NOTE: In this patient, the transection margin was assessed after removal of the specimen because of the proximity of the tumor to the neck of the pancreas. 
17. Divide the splenic vein.

NOTE: The vein should not be divided now if tumor proximity to the spleno-mesenteric junction poses concerns about the radicality of the procedure. In these instances, vein resection and reconstruction are required to achieve an R0 resection.

\section{Vein resection and reconstruction}

1. Plan for the most appropriate type of vein resection and prepare accordingly for reconstruction. If needed, identify a vascular segment suitable for reconstruction.

2. Achieve control of all vascular pedicles.

3. Cross-clamp the splenic vein upstream to the site of the tumor involvement.

4. Cross-clamp the superior mesenteric artery to reduce the amount of blood pooling in the intestines during venous cross-clamping

5. Cross-clamp the superior mesenteric vein and the portal vein.

6. Excise the involved venous segment en-bloc with the specimen. Carry out a side-wall resection of the portal-mesenteric junction. Harvest the inferior mesenteric vein. Place an e-PTFE suture between the inferior mesenteric vein graft and the upper corner of the vein defect.

7. If a vascular patch is needed for closure of the vascular defect, suture the vascular patch using two half-running sutures of $6 / 0$ e-PTFE.

8. Before releasing the clamps, flush the vein with saline solution containing sodium heparin using a ureteral catheter connected to a syringe. NOTE: The laparoscopic surgeon performs vascular flushing.

9. Remove the bulldog clamps. Remove first the bulldog on the portal vein to check for bleeding sites at a lower pressure.

\section{Completion of dissection}

1. Complete clearance of retroperitoneal vessels. Dissect along the periadvential plane of the superior mesenteric artery in a cephalad direction.

2. Skeletonize the right side of the superior mesenteric artery and remove the right celiac ganglion, if the tumor is located close to the neck of the pancreas, because of lymphatic drainage ${ }^{23}$ and path for neural invasion ${ }^{24}$.

3. Once the aortic plane is reached on the right side, perform the same dissection on the left side.

4. Complete the posterior dissection. Remove the left celiac ganglion en-bloc with the specimen. When using harmonic shears, pay attention to the active blade that is opposite to the artery. When finer dissection is required, use cold scissors.

5. Divide the short gastric vessels along the posterior surface and the upper margin of the pancreas.

6. Mobilize the spleen.

\section{Protection of retroperitoneal vessels}

1. Mobilize the round and falciform ligaments.

2. Cover the naked retroperitoneal vessels with round and falciform ligaments.

\section{Specimen extraction and wound closure}

1. Make a Pfannenstiel incision $(\sim 5 \mathrm{~cm})$.

2. Extract the specimen and load in an endoscopic bag.

3. Close the incision in layer and insufflate the abdomen for final exploration.

4. Close the fascia of the $12 \mathrm{~mm}$ assistant port.

5. Place a $14 \mathrm{Fr}$ pigtail catheter close to the pancreatic stump

6. Deflate the pneumoperitoneum.

7. Close all incisions.

\section{Representative Results}

The operation time was $6 \mathrm{~h}$ and $15 \mathrm{~min}$ with an estimated blood loss of $150 \mathrm{~mL}$. The time required to complete the vascular suture of the patch applied to the sidewall defect of the portomesenteric junction was $11 \mathrm{~min}$. The postoperative course was uneventful. Pathology demonstrated a moderately differentiated ductal adenocarcinoma of the pancreas $(\mathrm{G} 2 / 3)$, with perineural invasion and involvement of the spleno-mesenteric junction. All the 56 resected lymph nodes were negative. Circumferential tumor margins, assessed at $1 \mathrm{~mm}$, were also negative making the resection radical. The final pathology stage of this tumor was T3 NO RO. At the longest follow-up of 30 months, the patient is alive, well, and disease-free.

At our institution, a robot-assisted radical antegrade modular pancreatosplenectomy was performed in 20 patients. Admittedly, during the same period of time, other patients suitable for a minimally invasive approach received the same procedure using a laparoscopic technique without robotic assistance. This was not due to patient selection or surgeon preference but to the fact that the robot was not always timely available at the time of planned surgery, because of competition with either other procedures performed by our group (e.g., pancreatoduodenectomy) or procedures performed by other groups (e.g., urologic procedures). 
Briefly, all procedures were completed under robotic assistance, without conversions to open surgery, despite three patients required associated vascular procedures (Table 1). Namely, two patients required resection and reconstruction of the spleno-mesenteric junction, and one patient required resection of the celiac trunk (modified Appleby procedure). The mean operative time was 325 min \pm 88.6 min. Post-operative complications developed in 12 patients $(60 \%)$, being severe according to the Clavien-Dindo classification ${ }^{25}$ in 3 patients $(3 a=2 ; 3 b=1)(15 \%)$. There were no 90 -day or in-hospital deaths. Grade B post-operative pancreatic fistula ${ }^{26}$ developed in 5 patients (35\%). There was no grade $\mathrm{C}$ post-operative pancreatic fistula. Pathology demonstrated ductal adenocarcinoma in 14 patients, malignant intraductal papillary mucinous tumor in 5 patients, and pancreatic neuroendocrine cancer in one patient. In a patient population with a mean tumor diameter of $34 \mathrm{~mm} \pm 13 \mathrm{~mm}$, circumferential tumor margins, assessed at $1 \mathrm{~mm}$, were negative in 17 patients $(85 \%)$. The mean number of examined lymph nodes was $39 \pm$ 16.6.

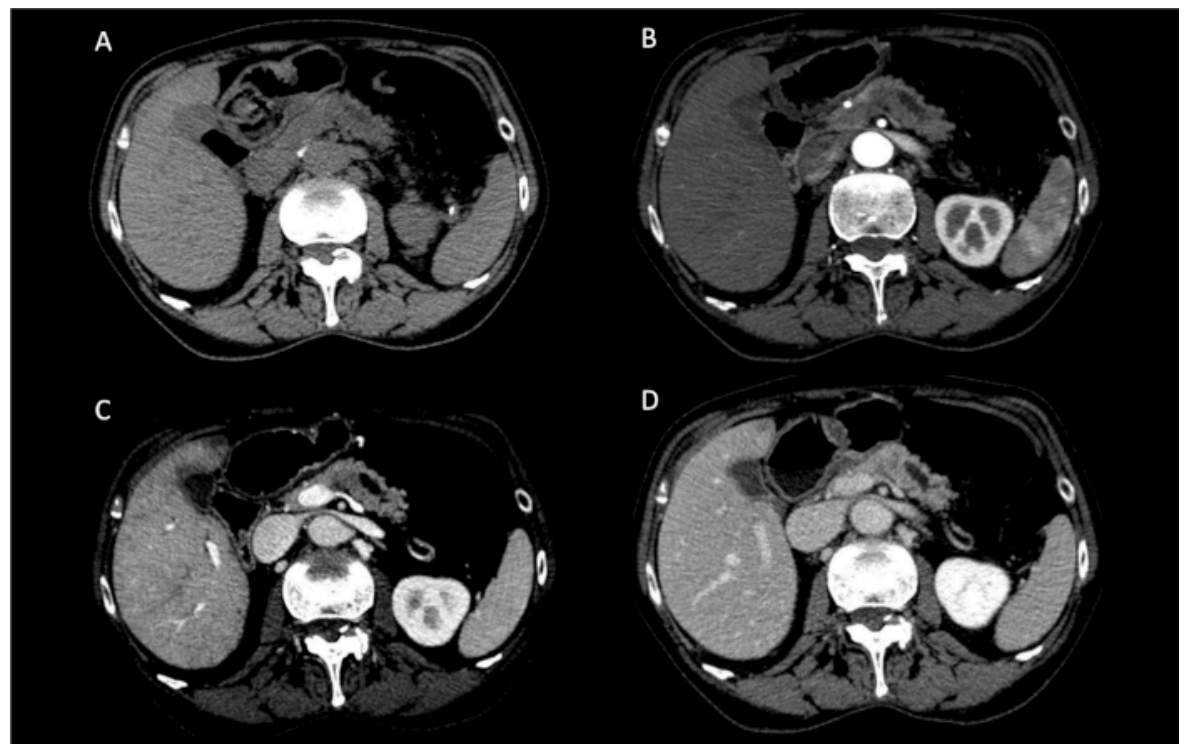

Figure 1: Preoperative computed tomography scan. (A) Basal; (B) Arterial phase; (C) Venous phase; (D) Parenchymal phase. A hypoenhancing pancreatic tumor, with upstream dilation of the pancreatic duct, is noted in the proximal part of the body of the pancreas. Please click here to view a larger version of this figure.

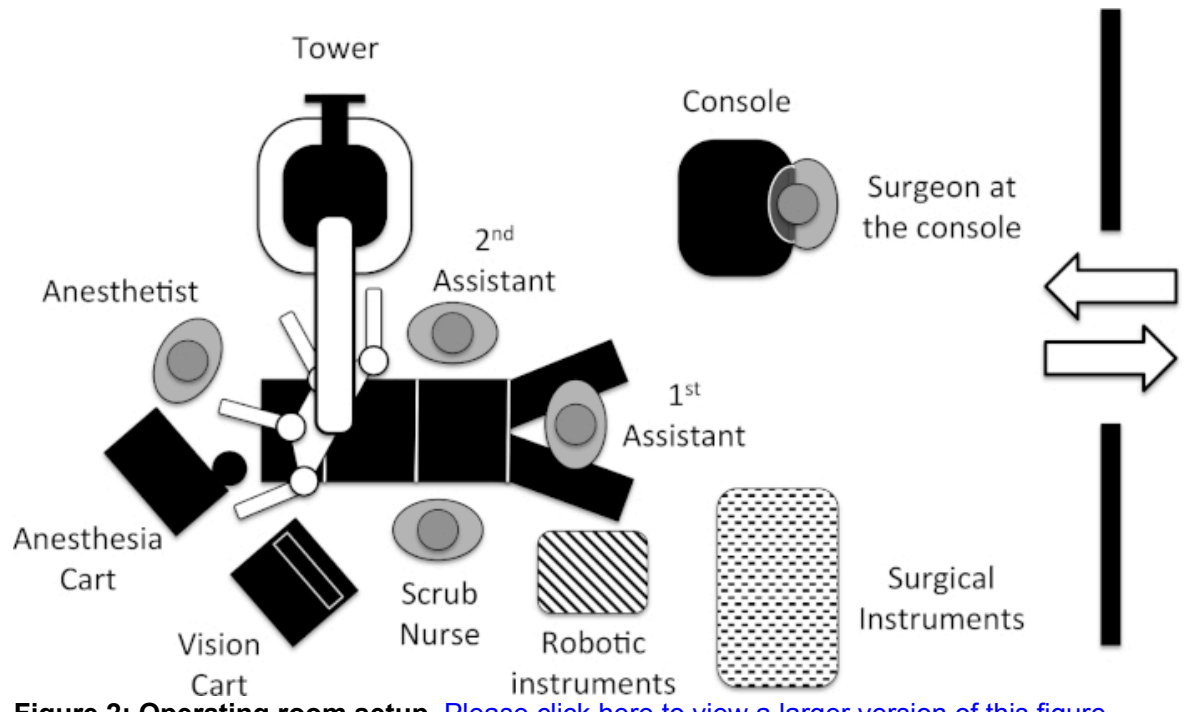

Figure 2: Operating room setup. Please click here to view a larger version of this figure. 

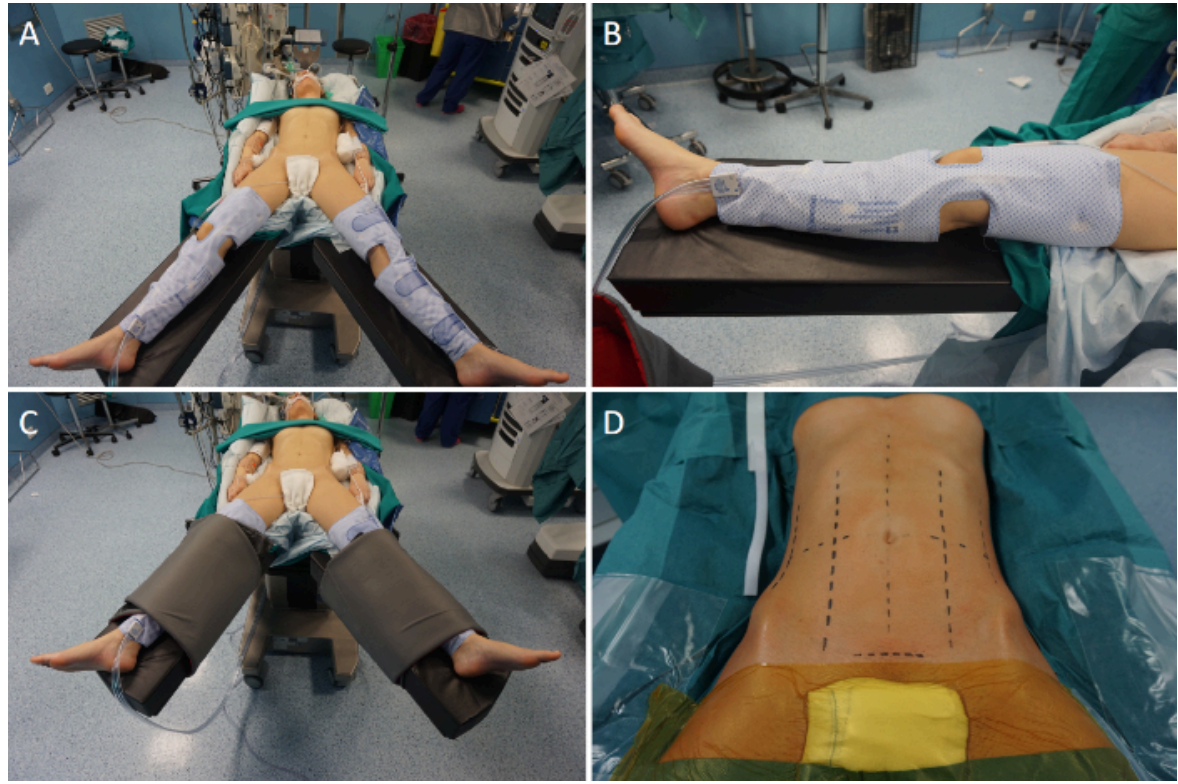

Figure 3: Operation setting. (A) The patient is placed supine with the legs parted. (B) Intermittent pneumatic compression cuffs are placed around the legs. (C) The patient is secured to the operating table using wide bandings. (D) The abdomen is prepped widely. Please click here to view a larger version of this figure.
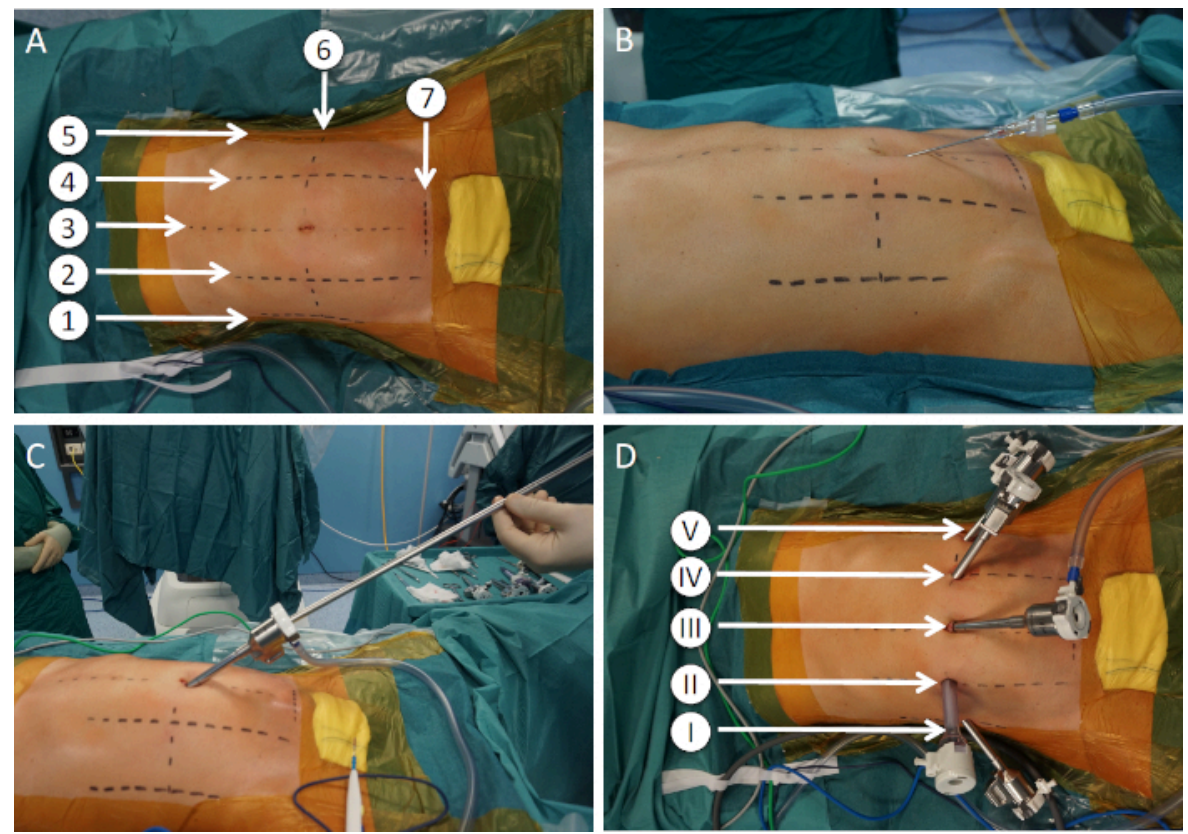

Figure 4: Port placement and extraction site. (A) Abdominal landmarks. 1: right anterior axillary line; 2: right pararectal line; 3 : midline; 4 left pararectal line; 5: left anterior axillary line; 6: transverse umbilical line; 7: suprabubic extraction site. (B) Pneumoperitoneum induction using a Veress needle technique. (C) Optic port placed immediately below the umbilicus. (D) Ports. I: robotic port for arm 1; II: assistant port; III: robotic port for arm 2 (optic); IV: robotic port for arm 3; V: robotic port for arm 4. Please click here to view a larger version of this figure. 


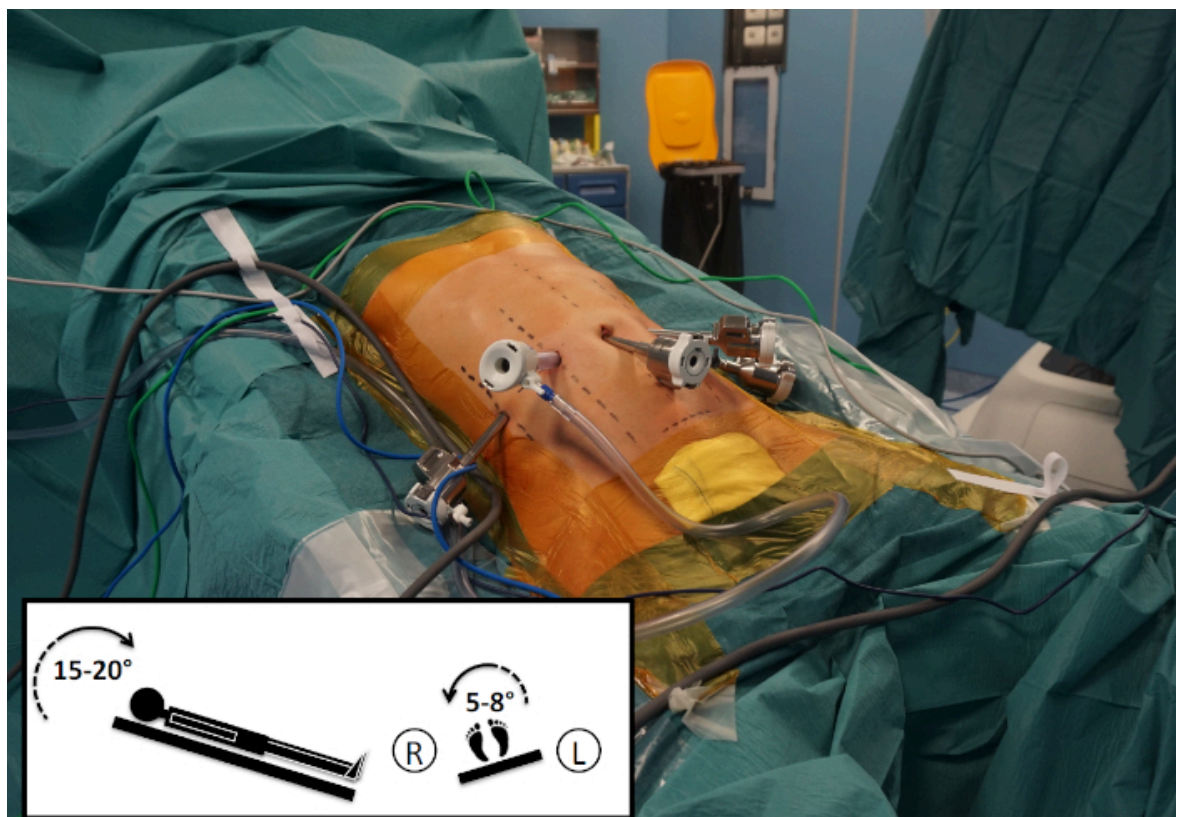

Figure 5: Operating table orientation. As highlighted in the square in the lower left corner, the operating table is oriented $15-20^{\circ}$ in reverse Trendelenburg and tilted $5-8^{\circ}$ to the patient's right side. Please click here to view a larger version of this figure.
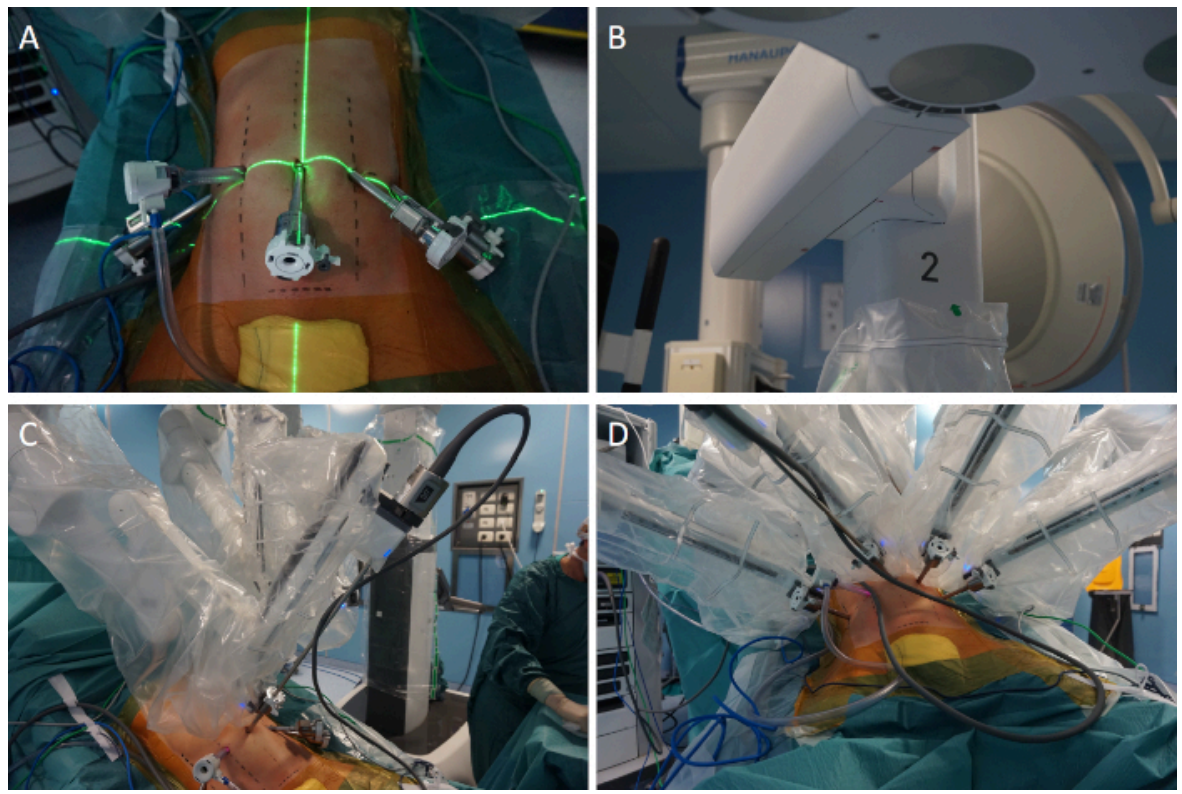

Figure 6: Docking of the surgical system for distal pancreatectomy. (A) Alignment of the laser crosshair of the boom over the initial camera port. (B) Direction of the camera arm (number 2) between $L$ and $E$ on the FLEX icon located at the base of the robotic arm. (C) Docking of the robotic arm 2 and insertion of the robotic camera. (D) After completion of targeting, the remaining arms are docked. Please click here to view a larger version of this figure. 


\begin{tabular}{|c|c|c|}
\hline & Mean or number & Standard deviation or percentage \\
\hline Operative time $(\min )$ & 325 & \pm 88.6 \\
\hline Associated vascular procedures & 3 & $15 \%$ \\
\hline Vein resection and reconstruction & 2 & $10 \%$ \\
\hline Arterial resection (modified Appleby procedure) & 1 & $5 \%$ \\
\hline Post-operative complications ${ }^{25}$ & 12 & $60 \%$ \\
\hline Severe post-operative complications ( $\geq$ grade 3 ) & 3 & $15 \%$ \\
\hline $\begin{array}{l}\text { Clinically relevant post-operative pancreatic } \\
\text { fistula }^{26}\end{array}$ & 5 & $25 \%$ \\
\hline Grade B post-operative pancreatic fistula & 5 & $25 \%$ \\
\hline Grade C post-operative pancreatic fistula & 0 & - \\
\hline 90-day or in-hospital mortality & 0 & - \\
\hline \multicolumn{3}{|l|}{ Tumor type } \\
\hline Ductal adenocarcinoma & 14 & $70 \%$ \\
\hline Malignant mucinous intraductal papillary tumor & 5 & $25 \%$ \\
\hline Neurondocrine carcinoma & 1 & $5 \%$ \\
\hline Tumor diameter (mm) & 34 & \pm 13 \\
\hline \multicolumn{3}{|l|}{ Tumor margins (assessed at $1 \mathrm{~mm}$ ) } \\
\hline Negative (R0) & 17 & $85 \%$ \\
\hline Examined lymph nodes & 39 & \pm 16.6 \\
\hline
\end{tabular}

Table 1: Results of 20 consecutive robot-assisted radical antegrade modular pancreatosplenectomies.

\section{Discussion}

Radical antegrade modular pancreatosplectomy aims at increasing the rate of radical resection for tumors located in the body and tail of the pancreas, as well as to achieve radical lymphoneurectomy. Depending on the degree of tumor growth in the retroperitoneum, the left adrenal gland can be either spared (anterior radical antegrade modular pancreatosplectomy) or removed en-bloc with the specimen (posterior radical antegrade modular pancreatosplectomy). In all procedures the Gerota fascia covering the upper pole of the left kidney must be removed as well as all the lympho-neural tissues surrounding the common hepatic artery, the celiac trunk, and the left aspect of the superior mesenteric $\operatorname{artery}^{11,27}$.

Overall radical antegrade modular pancreatosplectomy is a complex procedure even when using an open approach. Although radical antegrade modular pancreatosplectomy has also been performed using pure laparoscopic techniques ${ }^{12,28}$, the use of a robotic system is thought to facilitate the procedure due to the enhanced dexterity offered by robotic assistance ${ }^{29}$. Indeed, Duouadi et al. found that robotic assistance reduced the rate of conversion to open surgery while increasing the number of resected lymph nodes and the rate of margin negative resections ${ }^{13}$.

When the tumor is located close to the neck of the pancreas, involvement of the superior mesenteric-portal vein and/or the celiac trunk may occur, further complicating the procedure. Both arterial and venous resections have been performed using robotic assistance during radical antegrade modular pancreatosplectomy ${ }^{30}$, but the safety and oncologic efficacy of these procedures remain to be established.

In the case presented here, we performed a sidewall resection of the portomesenteric axis. The defect was closed using a vein patch. We still consider overt vascular involvement a contraindication to robotic approach ${ }^{18,31}$. However, we have performed a few robotic pancreatic resections with associated vascular procedures when vascular involvement was limited, and operative conditions permitted the procedure to be safely completed under robotic assistance ${ }^{32}$. We have already performed over 500 of such procedures open and we have experience with both pancreatic ${ }^{33}$ and renal ${ }^{34}$ robotic transplants.

Not all pancreatic tumors located in the body-tail of the pancreas can be resected using minimally invasive techniques, including roboticassistance. Although the contraindications to robotic resection are expected to vary with center and surgeon experience, it could be reasonable to accept that patients with truly locally advanced cancers, with portal hypertension secondary to superior mesenteric portal vein stenosis/ obstruction, with severe central obesity, and/or requiring multivisceral resections are less likely to be safely resected robotically than open.

Although current guidelines recommend upfront resection for pancreatic cancers not meeting the criteria to be classified either "borderline resectable" or "locally advanced" ${ }^{35}$, neoadjuvant treatments may also be beneficial in patients with immediately resectable tumors ${ }^{36,37}$. No evidence is currently available on the impact of the new neoadjuvant treatments on both the feasibility and safety of minimally invasive pancreatic resections. This issue is probably worth to be explored. 


\section{Disclosures}

The authors have nothing to disclose.

\section{Acknowledgments}

The authors have no acknowledgements.

\section{References}

1. Rahib, L. et al. Projecting cancer incidence and deaths to 2030: the unexpected burden of thyroid, liver, and pancreas cancers in the United States. Cancer Research. 74 (11), 2913-2921 (2014).

2. Rhim, A.D. et al. EMT and dissemination precede pancreatic tumor formation. Cell. 148 (1-2), 349-361 (2012).

3. Suker, M. et al. FOLFIRINOX for locally advanced pancreatic cancer: a systematic review and patient-level meta-analysis. Lancet Oncology. 17 (6), 801-810 (2016).

4. Hackert, T. et al. Locally Advanced Pancreatic Cancer: Neoadjuvant therapy with Folfirinox results in resectability in $60 \%$ of the patients Annals of Surgery. 264 (3), 457-463 (2016).

5. Conroy, T. et al. FOLFIRINOX or Gemcitabine as adjuvant therapy for pancreatic cancer. New England Journal of Medicine. 379 (25), 2395-2406 (2018).

6. Ling, Q., Xu, X., Zheng, S.S., Kalthoff, H. The diversity between pancreatic head and body/tail cancers: clinical parameters and in vitro models. Hepatobiliary \& Pancreatic Diseases International. 12 (5), 480-487 (2013).

7. Seufferlein, T., Bachet, J.B., Van Cutsem, E., Rougier, P.; ESMO Guidelines Working Group. Pancreatic adenocarcinoma: ESMO-ESDO clinical practice guidelines for diagnosis, treatment and follow-up. Annals of Oncology. 23 (Suppl 7), vii33-40 (2012).

8. Ghaneh, P. et al. The impact of positive resection margins on survival and recurrence following resection and adjuvant chemotherapy for pancreatic ductal adenocarcinoma. Annals of Surgery. 269 (3), 520-529 (2019).

9. Mirkin, K.A., Hollenbeak, C.S., Wong, J. Greater lymph node retrieval and lymph node ratio impacts survival in resected pancreatic cancer. Journal of Surgical Research. 220, 12-24 (2017).

10. Ling, Q. et al. The prognostic relevance of primary tumor location in patients undergoing resection for pancreatic ductal adenocarcinoma. Oncotarget. 8 (9), 15159-15167 (2017).

11. Strasberg, S.M., Drebin, J.A., Linehan, D. Radical antegrade modular pancreatosplenectomy. Surgery. 133 (5), $521-527$ (2003).

12. Kim, E.Y., Hong, T.H. Initial experience with laparoscopic radical antegrade modular pancreatosplenectomy for left-sided pancreatic cancer in a single institution: technical aspects and oncological outcomes. BMC Surgery. 17 (1), 2 (2017).

13. Daouadi, M. et al. Robot-assisted minimally invasive distal pancreatectomy is superior to the laparoscopic technique. Annals of Surgery. $\mathbf{2 5 7}$ (1), 128-132 (2013)

14. Gandy, R.C. et al. Refining the care of patients with pancreatic cancer: the AGITG Pancreatic Cancer Workshop consensus. The Medical Journal of Australia. 204 (11), 419-422 (2016).

15. Boeck, S., Stieber, P., Holdenrieder, S., Wilkowski, R., Heinemann, V. Prognostic and therapeutic significance of carbohydrate antigen 19-9 as tumor marker in patients with pancreatic cancer. Oncology. 70 (4), 255-264 (2006).

16. Hayman, A.V. et al. CA 19-9 nonproduction is associated with poor survival after resection of pancreatic adenocarcinoma. American Journal of Clinical Oncology. 37 (6), 550-554 (2014).

17. Amorese, G. Properative evaluation and anesthesia in minimally invasive surgery of the pancreas. In Minimally Invasive Surgery of the Pancreas. Edited by Boggi, U., 49-63, Springer-Verlag Italia S.r.I. Milan, Italy (2018).

18. Boggi, U. et al. Robotic-assisted pancreatic resections. World Journal of Surgery. 40 (10), 2497-506 (2016).

19. Napoli, N. et al. The learning curve in robotic distal pancreatectomy. Updates in Surgery. 67 (3), 257-264 (2015).

20. Hammerquist, R.J., Messerschmidt, K.A., Pottebaum, A.A., Hellwig, T.R. Vaccinations in asplenic adults. American Journal of Health-System Pharmacy. 73 (9), e220-228 (2016).

21. Javed, A.A. et al. Postoperative omental infarct after distal pancreatectomy: appearance, etiology management, and review of literature. Journal of Gastrointestinal Surgery. 19 (11), 2028-2037 (2015).

22. Emam, T.A., Cuschieri, A. How safe is high-power ultrasonic dissection? Annals of Surgery. 237 (2), 186-191 (2003).

23. Cesmebasi, A. et al. The surgical anatomy of the lymphatic system of the pancreas.Clinical Anatomy. 28 (4), $527-537$ (2015).

24. Tsuchikawa, T. et al. Detailed analysis of extra-pancreatic nerve plexus invasion in pancreatic body carcinoma analyzed by 50 consecutive series of distal pancreatectomy with en-bloc celiac axis resection. Hepatogastroenterology. 62 (138), 455-458 (2015).

25. Dindo, D., Demartines, N., Clavien, P.A. Classification of surgical complications: a new proposal with evaluation in a cohort of 6336 patients and results of a survey. Annals of Surgery. 240 (2), 205-213 (2004).

26. Bassi, C. et al. The 2016 update of the International Study Group (ISGPS) definition and grading of postoperative pancreatic fistula: 11 Years After. Surgery. 161 (3), 584-591 (2017).

27. Strasberg, S.M., Linehan, D.C., Hawkins, W.G. Radical antegrade modular pancreatosplenectomy procedure for adenocarcinoma of the body and tail of the pancreas: ability to obtain negative tangential margins. Journal of the American College of Surgeons. 204 (2), $244-249$ (2007)

28. Sunagawa, H., Harumatsu, T., Kinjo, S., Oshiro, N. Ligament of Treitz approach in laparoscopic modified radical antegrade modular pancreatosplenectomy: report of three cases. Asian Journal of Endoscopic Surgery. 7 (2), 172-174 (2014).

29. Ishikawa, N. et al. Robotic dexterity: evaluation of three-dimensional monitoring system and non-dominant hand maneuverability in robotic surgery. Journal of Robotic Surgery. 1 (3), 231-233 (2007).

30. Ocuin, L.M. et al. Robotic and open distal pancreatectomy with celiac axis resection for locally advanced pancreatic body tumors: a single institutional assessment of perioperative outcomes and survival. HPB. 18 (10), 835-842 (2016).

31. Napoli, N. et al. Indications, technique, and results of robotic pancreatoduodenectomy. Updates in Surgery. 68 (3), $295-305$ (2016). 
32. Kauffmann, E.F. et al. Robotic pancreatoduodenectomy with vascular resection. Langenbeck's Archives of Surgery. 401 (8), $1111-1122$ (2016).

33. Boggi, U. et al. Laparoscopic robot-assisted pancreas transplantation: First world experience. Transplantation. 93 (2), $201-206$ (2012).

34. Boggi, U. et al. Robotic renal transplantation: First European case. Transplant International. 24 (2), 213-218 (2011).

35. Tempero, M.A. et al. Pancreatic adenocarcinoma, version 2.2017, NCCN Clinical Practice Guidelines in Oncology. Journal of the National Comprehensive Cancer Network. 15 (8), 1028-1061 (2017).

36. Tienhoven, G.V. et al. Preoperative chemoradiotherapy versus immediate surgery for resectable and borderline resectable pancreatic cancer (PREOPANC-1): A randomized, controlled, multicenter phase III trial. Journal of Clinical Oncolology. 36 (18), LBA4002 (2018).

37. Motoi, F. et al. Randomized phase II/III trial of neoadjuvant chemotherapy with gemcitabine and S-1 versus upfront surgery for resectable pancreatic cancer (Prep-02/JSAP05). Japanese Journal of Clinical Oncology. 49 (2), 190-194 (2019). 